Año 7 | No 28| Octubre-diciembre, 2018

ISSN: 2305-5790

DOI: http://dx.doi.org/10.5377/farem.v0i28.7611

https://rcientificaesteli.unan.edu.ni

\title{
Influencia de la imagen corporal en la práctica excesiva de ejercicios físicos en adolescentes de Estelí, Nicaragua
}

\section{Influence of the physical image in the excessive practice of physical exercises by teenagers of Estelí, Nicaragua}

\author{
Gissell Beatriz Aráuz Castillo ${ }^{1}$ \\ gissellcastillo@yahoo.com \\ Karina Dávila Montiel ${ }^{1}$ \\ karinadavila431@yahoo.com \\ Yaritza Daniela Acuña Flores' \\ floresyarialexan@yahoo.com \\ Franklin Solís Zúniga² \\ franksolis23@yahoo.com
}

Recibido: 15 de agosto de 2018, Aceptado: 06 de diciembre de 2018

\section{RESUMEN}

La influencia que tiene la imagen corporal en la población de adolescentes, es una temática poco indagada, a pesar de ser esta relevante ante la sociedad, abarca distintas áreas de conocimiento especialmente desde la psicología. El presente escrito, se enmarca en una investigación realizada con 32 adolescentes entre las edades de 14 y 17 años, siendo escogidos 8 adolescentes por cada 4 gimnasios con mayor afluencia en Estelí período 2017. El propósito fue analizar cómo influye la percepción de la imagen corporal en la práctica de ejercicios físicos excesivos, en adolescentes. El enfoque metodológico fue mixto con prevalencia cualitativa. Las técnicas utilizadas para la recolección de la información fueron: Entrevista, técnica de listado libre, test de autoestima y escala de Likert. Los principales hallazgos muestran que los adolescentes se ven influidos por la percepción de su propia imagen corporal, realizando excesivamente ejercicios físicos, siendo afectados por factores como: la sociedad, autoestima, estereotipos de género que se difunden erróneamente, dedicando más de 2 horas a la práctica de ejercicios donde descuidan otras actividades que solían hacer. A partir de los resultados de esta investigación se realizó como sugerencia un plan de intervención psicológica que pueda ser utilizado por siguientes investigadores para trabajar con entrenadores y adolescentes practicantes del ejercicio físico excesivo para prevenir el trastorno dismórfico corporal o también conocido popularmente como vigorexia.

Palabras claves: percepción; imagen corporal; factores; vigorexia.

1 Egresada de la Licenciatura en Psicología. UNAN-Managua/FAREM-Estelí.

2 Docente UNAN-Managua/FAREM-Estelí.

(C) 2018 - Revista Científica de FAREM-Estelí. 


\section{ABSTRACT}

The influence that body image has on the young population is not a popular researched subject, although it is significant for the society, it covers different knowledge areas especially the psychological one. This article is part of a research carried out with 32 teenagers among 14 and 17 years old. 8 teenagers were chosen every 4 gyms with the highest affluence in Estelí during 2017. The aim of this research was to analyze how the perception of body image influences the practice of excessive physical exercises in teenagers. The methodological approach was mixed with qualitative approach incidence. To collect the data an Interview, free listing technique, self-esteem test and Likert scale were used. The main findings show that teenagers are influenced by the perception of their own physique image, performing excessively physical exercises, being affected by factors such as: society, self-esteem, gender stereotypes that extent erroneously, spending more than 2 hours daily to work, changing their routine. Based on the main findings, a psychological intervention plan was developed as an option to be used by future researchers to work with trainers and teenagers who work out, in order to prevent corporal dysmorphic disorder or popularly known as vigorexia.

Keywords: perception; body image; factors; vigorexia.

\section{INTRODUCCIÓN}

En la actualidad la preocupación por la imagen corporal, cada vez se incrementa, sobre todo en adolescentes en pleno desarrollo, mostrando estos que la imagen corporal es de suma importancia tanto para ellos como para la sociedad.

La inquietud por tener una imagen corporal perfecta ha influido para que muchas personas asistan a los gimnasios a realizar ejercicios físicos excesivos, notando una distorsión cognitiva de su propia imagen al no sentirse bien consigo mismos.

\section{Según Frozzotti (2015)}

Los estereotipos de belleza impuestos a través de los medios de comunicación y las respuestas de la población hacen frente a aquellos modelos culturales, que se asumen al consumismo, los cuales muchas veces muestran la delgadez extrema como sinónimo de belleza.

En los hallazgos de la investigación Estudio de la satisfacción de la imagen corporal en la educación primaria, relaciones con la actividad física y nivel de condición de los escolares, entre sus resultados se encontró que en edades tempranas el alumnado presenta niveles más bajos de insatisfacción corporal que en edades posteriores (Aviles, 2013 - 2015).

Por otra parte, en la investigación Influencia de modelos ideales de belleza y delgadez, propuesto por los medios de comunicación, en adolescentes de 15 a 18 años, de la Provincia de Buenos Aires durante el período 2013, los resultados explican que adolescentes de 15 a 16 años, poseen una influencia aún más directa de los estereotipos de belleza y delgadez, teniendo como conclusión que todas las adolescentes, de 15 a 18 años de la provincia de Buenos Aires, existe una influencia de las imágenes difundidas por los medios de comunicación, mediante publicidades, así como también una autoevaluación de la propia imagen corporal y la existencia de conductas relacionadas a la reducción del peso, que tienen una relación directa con los modelos estéticos socialmente aceptados (Balbi, 2013, pág. 78)

El estudio, Prevalencia de conductas alimentarias de riesgo y trastornos alimentarios en adultos jóvenes, en sus principales resultados encontraron que del $5 \%$ adultos jóvenes son con conductas alimentarias de riesgo, el $22 \%$ de adultos jóvenes con riesgo de presentar conductas alimentarias de riesgo y un $73 \%$ de adultos jóvenes no presentan estás conductas (Vinueza, 2015).

En cuanto a la investigación Manifestaciones del trastorno vigorexia, presentes en usuarios de gimnasio del municipio, En sus principales resultados encontraron que un $25 \%$, de los hombres encuestados, no están catalogados como vigoréxicos, podrían estar propensos a desarrollar el trastorno, debido a que presentan las mayorías de las manifestaciones, en cuanto a las mujeres a un $9 \%$ de ellas se le puede hacer los señalamientos anteriores (Kishory, 2010). 
Arróliga Paz, López Moreno, \& Rodríguez Moreno, (2010); realizaron la investigación denominada Factores Psicosociales asociados a la aparición de conductas vigoréxicas en los jóvenes que asisten a los gimnasios de la ciudad de Estelí, Nicaragua y los hallazgos determinan que factores psicosociales asociados a la aparición de conductas vigoréxicas en los jóvenes que asisten a los principales gimnasios son: baja autoestima, insatisfacción con el propio cuerpo, influencia de amigos y medios de comunicación, los cual los lleva a la práctica excesiva de ejercicios físicos.

\section{MATERIALES Y MÉTODOS}

\section{Tipo de estudio}

La investigación se realizó a partir de un diseño mixto, ajustando distintas técnicas cuantitativas y cualitativas. Para fines del estudio se seleccionaron a cuatro gimnasios más reconocidos y con mayor afluencia de la ciudad de Estelí.

\section{Población y muestra}

La población que se estudió, fueron adolescentes que asisten a los gimnasios más reconocidos y con mayor afluencia que son: gimnasio Guido, Gold's Gym, Strong Gym y United Fitness. La muestra estuvo conformada por 32 adolescentes, 8 por cada uno de los gimnasios 4 del sexo femenino y 4 del sexo masculino. Se eligieron de esta manera porque cumplían la mayor parte de los criterios de inclusión. El proceso de selección de la muestra con los adolescentes, se realizó mediante el muestreo no probabilístico, de tipo casual, ya que se requería cumplir con los siguientes criterios:

\section{Criterios de inclusión}

- Adolescentes entre las edades de 14 a 17 años

- Sean adolescentes activos en la práctica de ejercicios físicos dentro y fuera de los gimnasios

- Igual número de participación por sexo (Hombres y mujeres)

\section{Métodos y técnicas para la recolección de datos}

Para la recolección de datos se aplicaron las técnicas de la entrevista, listado libre, test de autoestima y escala de Likert. La entrevista constó con 10 ítems enfocados a conocer la percepción que tienen los adolescentes a cerca de la imagen corporal, pero se escogieron 4 de estos ítems como los más relevantes. El listado libre constó de 5 palabras que asociadas a la imagen corporal que demostraron los adolescentes dentro de sus explicaciones las percepciones hacia esta palabra generadora.

El test de autoestima constó de 40 Ítems que estado actual correspondiente a la autoestima de los adolescentes.

La escala de Likert con sus 31 ítems ayudo a obtener información de los adolescentes a cerca de sus actitudes entre otras cosas más.

\section{Procesamiento y análisis de datos}

Una vez que se obtuvieron los datos, estos fueron analizados según su naturaleza ya siendo cualitativa o cuantitativa. Para la entrevista, se realizó una transcripción fiel de la información obtenida posteriormente se hizo una síntesis y análisis de los datos utilizando el programa Microsoft Word.

Para el listado libre se analizó cuantitativa como cualitativamente donde las respuestas que eran establecidas relacionadas a la percepción de la imagen corporal, rol de género y factores, siendo analizadas por medio de Microsoft Excel y Microsoft Word.

El test de autoestima se analizó de acuerdo a las normas evaluativas de validez y confidencialidad estipuladas por este para su aplicación y revisión; posteriormente se agregó a la base de datos del programa estadístico SPSS, donde se relacionaron estadísticamente la variables autoestima y el ítem de la escala de Likert Me preocupa la opinión de los demás en cuanto a mi apariencia y el Nivel de autoestima de los adolescentes a través de pruebas de Chi- cuadrada, mostrando grafico de barras y tabla cruzada. Esto fue analizado cuantitativamente.

Los 31 ítems de la escala se añadieron a la base de datos de SPSS el cual se obtuvo una tabla de frecuencia y porcentaje, pero posteriormente se realizó un 
resumen de eso en una tabla con los ítems de mayor cantidad para los factores y también siendo utilizada para las actitudes que los adolescentes presentan.

Los adolescentes, objetos de estudio, revelaron que la imagen corporal es verse bien más cuando se habla del físico, además, piensan que el físico es importante para la vida diaria, ya que les hace sentirse bien con ellos mismos.

La mayoría piensa que realizar ejercicios físicos es importante para la salud, ya que fortalece los huesos y músculos día a día, además algunos piensan que es importante para tener un cuerpo atractivo, ponerte en forma y por qué es una manera de distracción para los que practican, también que estos piensan que el sexo opuesto se fijaría más en ellos por la mejora que va obteniendo su cuerpo.

\section{RESULTADOS Y DISCUSIÓN}

Percepciones que tienen las y los adolescentes acerca de su imagen corporal

En los adolescentes se mostró que hay una motivación, que hace que ellos realicen ejercicios físicos, ya que algún miembro de su familia o amigos lo realiza y lo desempeñan, por lo tanto, es una forma que influye a querer ser y verse como ellos.

Aunque especulan que, si tienen la rutina adecuada y que el peso que realizan va de acuerdo a su cuerpo, afirmando conocer que si exceden el peso puede tener problemas de salud, como dañar su cuerpo en lugar de mejorarlo.

A continuación, se muestra el siguiente diagrama que se alude a los resultados de la percepción de la imagen corporal de los adolescentes:

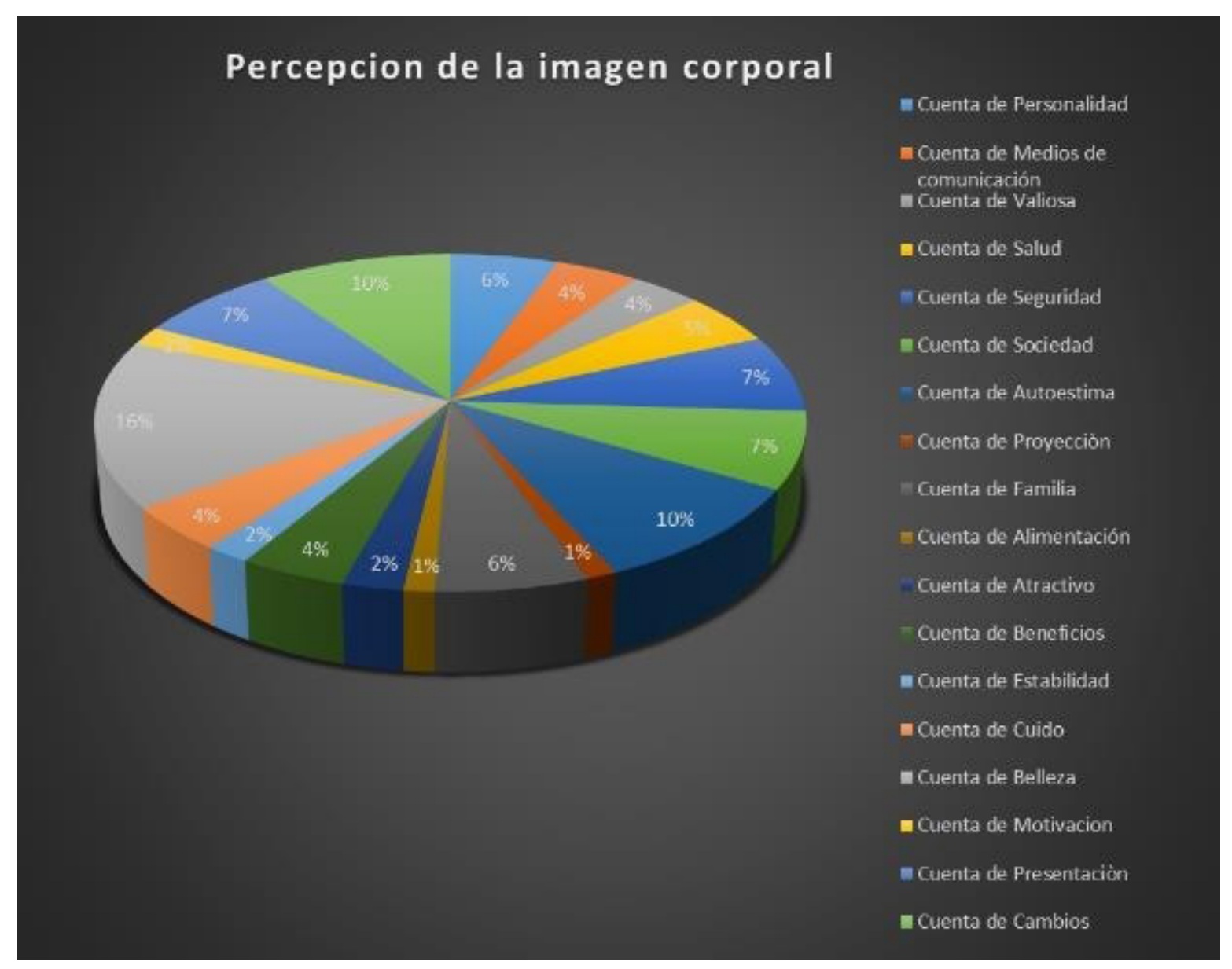


Dentro de este diagrama se pueden ver los resultados cuantitativos en base a la percepción de la imagen corporal a continuación se explica mejor:

Un $16 \%$ belleza, $10 \%$ cambios e igual con un $10 \%$ autoestima, $7 \%$ sociedad e igual se tiene con un $7 \%$ seguridad, $7 \%$ personalidad, seguidamente con un $6 \%$ presentación, $6 \%$ familia, con un $5 \%$ salud, $4 \%$ comunicación e igual con un $4 \%$ valiosa, $4 \%$ cuido y $4 \%$ beneficio, un $2 \%$ estabilidad igualándose con un $2 \%$ de la palabra atractivo, con un $2 \%$ motivación, con un $1 \%$ proyección, con un $1 \%$ alimentación.

Es importante resaltar que los pensamientos que estos tienen son creencias que a través de estímulos externos y de manera consciente construyan en la mente la imagen corporal perfecta estereotipada.

Factores que influyen en la percepción de la imagen corporal en los adolescentes que practican ejercicio físico excesivo

En la presente tabla cruzada describe los niveles de autoestima con la variable, Me preocupa la opinión de los demás en cuanto a mi apariencia, en esta se reflejan los mayores porcentajes por cada una y el número de adolescentes que se refirió a esto.

Tabla cruzada

\begin{tabular}{|c|c|c|c|c|c|c|c|}
\hline & & & \multicolumn{4}{|c|}{ Nivel de autoestima de los adolescentes } & \multirow[b]{2}{*}{ Total } \\
\hline & & & $\begin{array}{c}\text { Autoestima } \\
\text { alta } \\
\text { negativa }\end{array}$ & $\begin{array}{c}\text { Autoestima } \\
\text { alta } \\
\text { positiva }\end{array}$ & $\begin{array}{c}\text { Autoestima } \\
\text { baja } \\
\text { positiva }\end{array}$ & $\begin{array}{c}\text { Autoestima } \\
\text { baja } \\
\text { negativa }\end{array}$ & \\
\hline \multirow{12}{*}{$\begin{array}{l}\text { Me preocupa } \\
\text { la opinión de } \\
\text { los demás en } \\
\text { cuanto a mi } \\
\text { apariencia }\end{array}$} & \multirow{3}{*}{$\begin{array}{l}\text { Muy de } \\
\text { acuerdo }\end{array}$} & Recuento & 0 & 5 & 3 & 1 & 9 \\
\hline & & $\begin{array}{l}\text { \% dentro de } \\
\text { Me preocupa la } \\
\text { opinión de los } \\
\text { demás en cuanto } \\
\text { a mi apariencia }\end{array}$ & $0.0 \%$ & $55.6 \%$ & $33.3 \%$ & $11.1 \%$ & $100.0 \%$ \\
\hline & & $\%$ del total & $0.0 \%$ & $15.6 \%$ & $9.4 \%$ & $3.1 \%$ & $28.1 \%$ \\
\hline & \multirow[t]{3}{*}{ De acuerdo } & Recuento & 0 & 2 & 1 & 6 & 9 \\
\hline & & $\begin{array}{l}\text { \% dentro de } \\
\text { Me preocupa la } \\
\text { opinión de los } \\
\text { demás en cuanto } \\
\text { a mi apariencia }\end{array}$ & $0.0 \%$ & $22.2 \%$ & $11.1 \%$ & $66.7 \%$ & $100.0 \%$ \\
\hline & & $\%$ del total & $0.0 \%$ & $6.3 \%$ & $3.1 \%$ & $18.8 \%$ & $28.1 \%$ \\
\hline & \multirow{3}{*}{$\begin{array}{l}\text { Ni de } \\
\text { acuerdo } \\
\text { ni en } \\
\text { desacuerdo }\end{array}$} & Recuento & 2 & 3 & 4 & 0 & 9 \\
\hline & & $\begin{array}{l}\text { \% dentro de } \\
\text { Me preocupa la } \\
\text { opinión de los } \\
\text { demás en cuanto } \\
\text { a mi apariencia }\end{array}$ & $22.2 \%$ & $33.3 \%$ & $44.4 \%$ & $0.0 \%$ & $100.0 \%$ \\
\hline & & $\%$ del total & $6.3 \%$ & $9.4 \%$ & $12.5 \%$ & $0.0 \%$ & $28.1 \%$ \\
\hline & \multirow{3}{*}{$\begin{array}{l}\text { Muy en } \\
\text { desacuerdo }\end{array}$} & Recuento & 0 & 1 & 3 & 1 & 5 \\
\hline & & $\begin{array}{l}\text { \% dentro de } \\
\text { Me preocupa la } \\
\text { opinión de los } \\
\text { demás en cuanto } \\
\text { a mi apariencia }\end{array}$ & $0.0 \%$ & $11.1 \%$ & $33.3 \%$ & $11.1 \%$ & $55.6 \%$ \\
\hline & & $\%$ del total & $0.0 \%$ & $3.1 \%$ & $9.4 \%$ & $3.1 \%$ & $15.6 \%$ \\
\hline
\end{tabular}




\begin{tabular}{|c|c|c|c|c|c|c|}
\hline \multirow[t]{3}{*}{ Total } & Recuento & 2 & 11 & 11 & 8 & 32 \\
\hline & $\begin{array}{l}\text { \% dentro de } \\
\text { Me preocupa la } \\
\text { opinión de los } \\
\text { demás en cuanto } \\
\text { a mi apariencia }\end{array}$ & $6.3 \%$ & $34.4 \%$ & $34.4 \%$ & $25.0 \%$ & $100.0 \%$ \\
\hline & $\%$ del total & $6.3 \%$ & $34.4 \%$ & $34.4 \%$ & $25.0 \%$ & $100.0 \%$ \\
\hline
\end{tabular}

En la tabla anterior se muestra el cruce de variables, Me preocupa la opinión de los demás en cuanto a mi apariencia y Nivel de autoestima de los adolescentes.

Se obtuvo como resultados, que existe un mayor porcentaje de autoestima baja negativa de $66.7 \%$ que equivale a 6 personas, en segundo lugar con un $60.0 \%$ con autoestima baja positiva que equivale a 3 adolescentes, en tercer lugar con un $55.6 \%$ de autoestima alta positiva que equivale a 5 adolescentes, $44.4 \%$ de autoestima baja positiva que equivale a 4 adolescentes, con un $34.4 \%$ de autoestima alta positiva y baja negativa que equivale a 11 adolescentes con autoestima alta positiva y 11 adolescentes con autoestima baja negativa, 33.3\% con autoestima baja positiva que equivale a 3 adolescentes, así mismo con el mismo porcentaje con autoestima alta positiva de igual manera equivale a 3 adolescentes.

Con un $25.0 \%$ con autoestima baja negativa que equivale a 8 adolescentes, $22.2 \%$ con autoestima alta negativa y alta positiva que equivale a 2 adolescentes cada uno, con un $20.0 \%$ con autoestima alta positiva y autoestima baja negativa que equivale a 1 personas para cada uno. Así mismo $11.1 \%$ con autoestima baja negativa y baja positiva que equivale a 1 adolescentes. Y con un mínimo porcentaje de $6.3 \%$ de autoestima alta negativa que equivale a 2 personas.
La presente tabla explica detalladamente las pruebas de Chi-cuadrado que se constata con la tabla cruzada anterior, esta muestra los valores, grados de libertad y la significancia.

Pruebas de Chi-cuadrada

\begin{tabular}{lrrr}
\hline & Valor & Gl & \multicolumn{2}{c}{$\begin{array}{c}\text { Sig. asintótica } \\
\text { (2 caras) }\end{array}$} \\
\hline $\begin{array}{l}\text { Chi-cuadrado de } \\
\begin{array}{l}\text { Pearson } \\
\text { Razón de } \\
\text { verosimilitud }\end{array}\end{array}$ & $18.861 \mathrm{a}$ & 9 & .026 \\
$\begin{array}{l}\text { Asociación lineal } \\
\text { por lineal }\end{array}$ & .002 & 1 & .021 \\
N de casos válidos & 32 & 9 & .967 \\
\hline
\end{tabular}

a. 16 casillas (100.0\%) han esperado un recuento menor que 5. El recuento mínimo esperado es .31.

Dado como resultado por las variables: Me preocupa la opinión de los demás en cuanto a mi apariencia y el Nivel de autoestima de los adolescentes. En esta se refleja una significancia estadística entre las variables mencionadas. Al comprobarla se dice que el valor de x2 es de 18.861a que es un valor mayor que 3.84; siendo significativo al nivel de 0.05 afirmándose que los adolescentes se ven inducidos por el nivel de autoestima, el cual les preocupa la opinión de los demás en cuanto a su apariencia. 


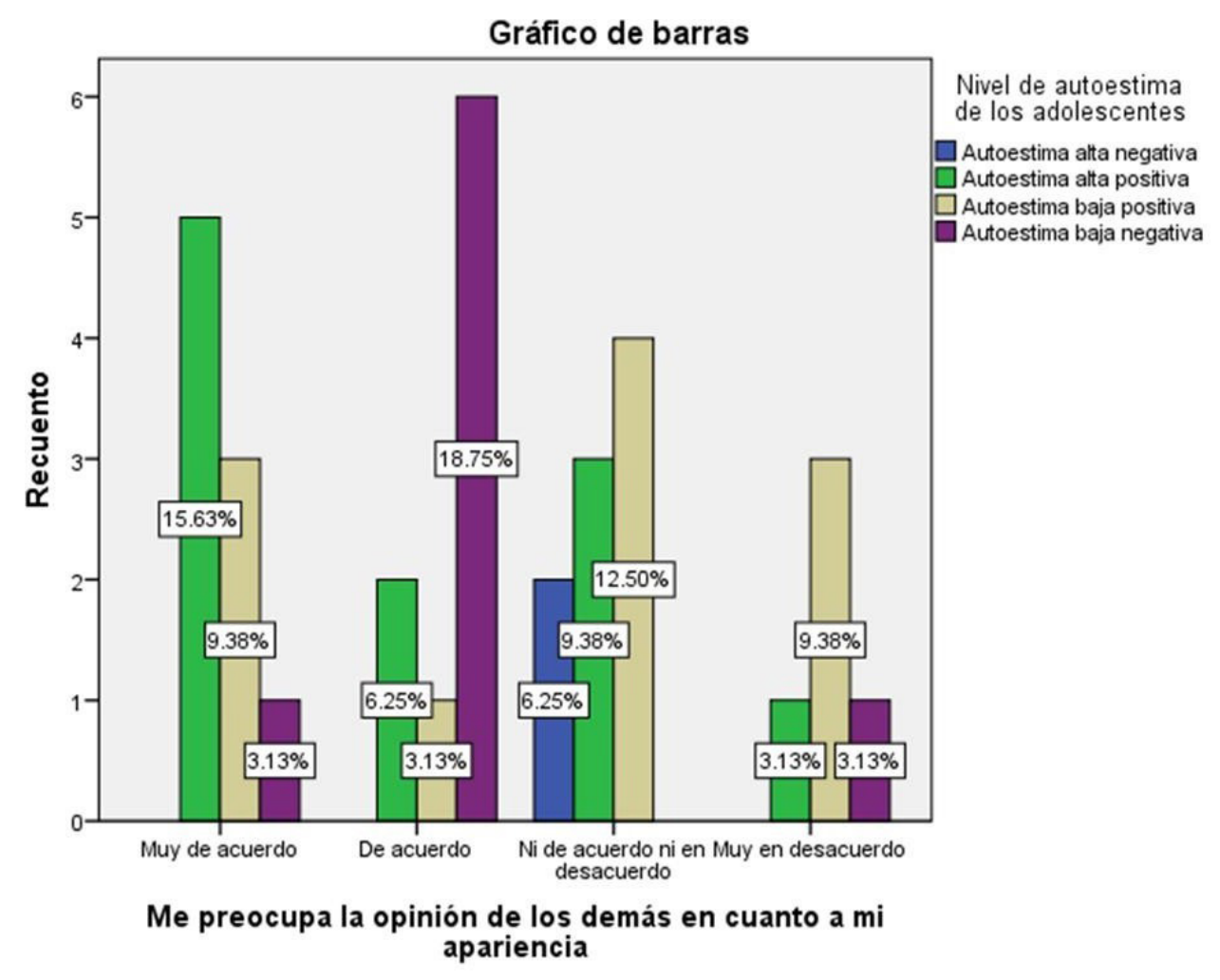

El gráfico de barras se constata por la tabla cruzada y la prueba de Chi-cuadrada, a continuación, se muestra el análisis cuantitativo:

Este gráfico muestra en escala las variables: Me preocupa la opinión de los demás en cuanto a mi apariencia y la variable Nivel de autoestima de los adolescentes, dando como resultado en los adolescentes su mayoría están de acuerdo con este ítems, dando como mayor porcentaje $18.75 \%$ con nivel de autoestima baja negativa, con un $15.63 \%$ cuenta con un nivel de autoestima alta positiva que están muy de acuerdo, así mismo con un $12.50 \%$ con un nivel de autoestima baja positiva que están ni de acuerdo ni en desacuerdo.

Con un nivel de $9.38 \%$ cuentan con un nivel de autoestima baja positiva y alta positiva donde los adolescentes están muy en desacuerdo, ni de acuerdo ni en desacuerdo y muy de acuerdo. Con un $6.25 \%$ cuenta con un nivel de autoestima alta negativa y alta positiva. Y con un mínimo porcentaje de $3.13 \%$ con un nivel de autoestima naja negativa y baja positiva y alta positiva.

De dicho procedimiento antes explicado se obtuvieron pruebas que mostraron el cruce de variables al que se le había sometido, donde es notable que existe un conflicto de los adolescentes en cuanto a su autoestima puesto que estos poseen en su mayoría, una baja autoestima negativa, lo cual esto hace que se genere una preocupación por lo que los demás opinen de su apariencia generando una inquietud en los mismos por ende estos terminan realizando ejercicios físicos excesivos para lograr la aceptación social y tener el cuerpo ideal estipulado y difundido por los medios de comunicación de masas.

Por medio del listado libre se obtuvo un diagrama que muestra los factores que influyen en la percepción de la imagen corporal a continuación se presenta: 


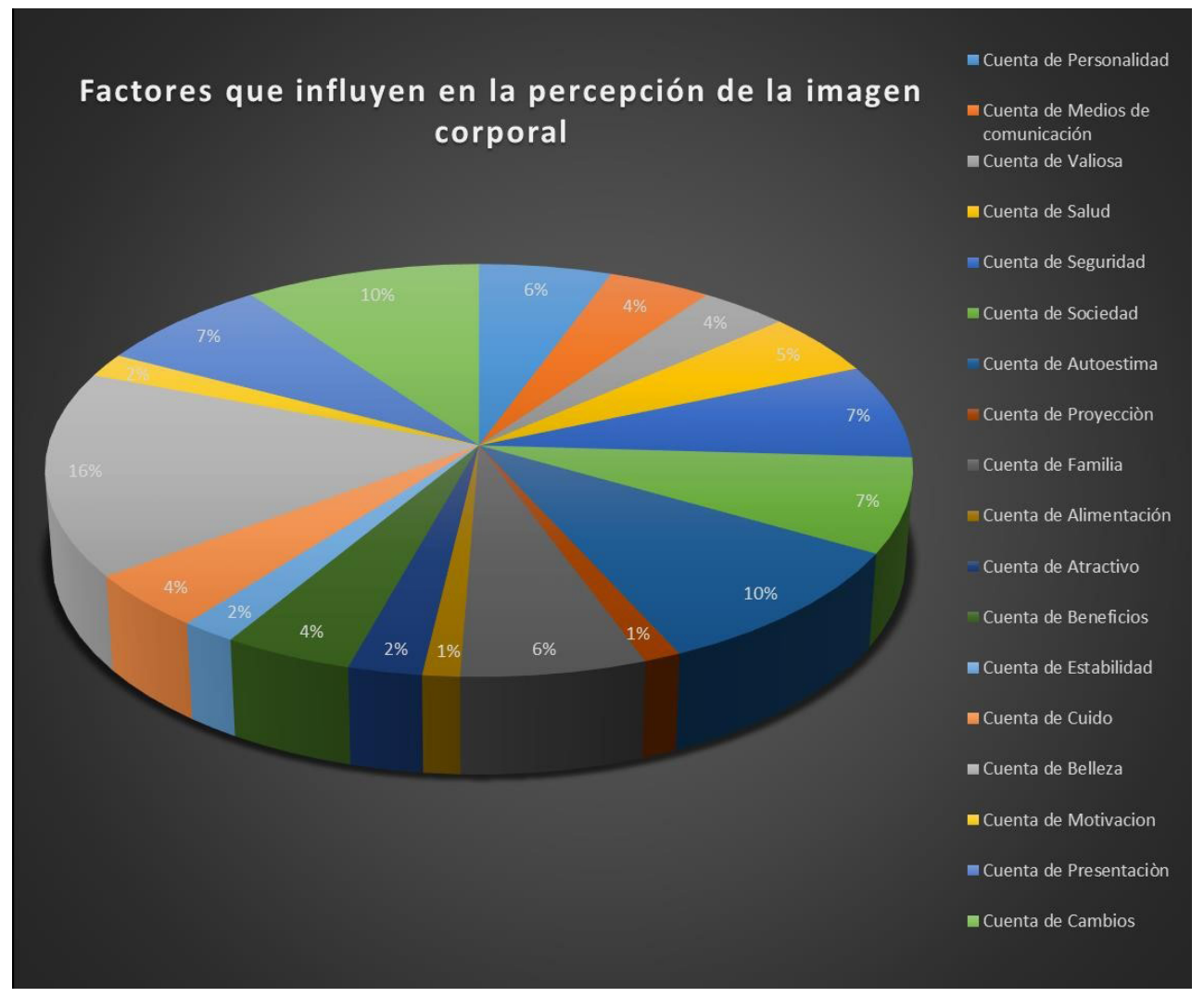

Análisis cuantitativo del diagrama factores:

Un $16 \%$ belleza, $10 \%$ cambios e igual con un $10 \%$ autoestima, $7 \%$ sociedad e igual se tiene con un $7 \%$ seguridad, $7 \%$ personalidad, seguidamente con un $6 \%$ presentación, $6 \%$ familia, con un $5 \%$ salud, $4 \%$ comunicación e igual con un $4 \%$ valiosa, $4 \%$ cuido y $4 \%$ beneficio, un $2 \%$ estabilidad igualándose con un $2 \%$ de la palabra atractivo, con un $2 \%$ motivación, con un $1 \%$ proyección, con un $1 \%$ alimentación.

Se puede observar que los factores que más influyen en la percepción de la imagen corporal tiene que ver con la sociedad siendo uno de los factores que influyen a que los adolescentes no se sientan conformes consigo mismos y de esta manera tiendan a modificar su cuerpo con ese deseo pertenecer a un grupo social y de ver cambios en su cuerpo, el cual no se dan cuenta de que se les está generando una distorsión cognitiva de la imagen corporal.
Es por ello que estos buscan a mejorarlo y al no observar eso que ellos quieren tener, terminan por asistir excesivamente a los gimnasios.

Actitudes que presentan los adolescentes ante la práctica de ejercicios físicos excesivos

En la presente escala se muestran los principales ítems que tienen mayor porcentaje respecto a las actitudes que presentan los adolescentes ante la práctica de ejercicios físicos excesivos.

\section{Escala de likert}

Clave:

MD (Muy de acuerdo) DA (De acuerdo) I (Ni de acuerdo ni en desacuerdo) ED (En desacuerdo) MED (Muy en desacuerdo). 
Tabla 1: Escala de Likert con resultados de las tablas de frecuencia y porcentaje obtenidas del programa SPSS

\begin{tabular}{|c|c|c|c|c|c|c|c|c|c|c|c|}
\hline & & \multicolumn{2}{|c|}{ MD } & \multicolumn{2}{|c|}{ DA } & \multicolumn{2}{|c|}{ I } & \multicolumn{2}{|c|}{ ED } & \multicolumn{2}{|c|}{ MED } \\
\hline & & $\mathbf{F}$ & $\mathbf{P}$ & $\mathbf{F}$ & $\mathbf{P}$ & $\mathbf{F}$ & $\mathbf{P}$ & $\mathbf{F}$ & $\mathbf{P}$ & $\mathbf{F}$ & $\mathbf{P}$ \\
\hline 1 & Realizo mis rutinas moderadamente & 12 & 37,5 & 12 & 37,5 & 6 & 18,8 & 0 & 0 & 2 & 6,3 \\
\hline 2 & $\begin{array}{l}\text { Realizo más de } 2 \text { horas de rutina en el } \\
\text { gimnasio. }\end{array}$ & 5 & 15,6 & 20 & 62,5 & 7 & 21,9 & 0 & 0 & 0 & 0 \\
\hline 3 & $\begin{array}{l}\text { Comparo mi figura estando con otras } \\
\text { personas. }\end{array}$ & 3 & 9,4 & 8 & 25,0 & 10 & 31,3 & 7 & 21,9 & 4 & 12,5 \\
\hline 4 & Siento que necesito ir al gimnasio. & 18 & 56,3 & 13 & 40,6 & 1 & 3,1 & 0 & 0 & 0 & 0 \\
\hline 5 & $\begin{array}{l}\text { Estoy dispuesto a asistir todo el día al } \\
\text { gimnasio. }\end{array}$ & 10 & 31,3 & 19 & 59,4 & 1 & 3,1 & 2 & 6,3 & 0 & 0 \\
\hline 6 & Realizo rutina en el gimnasio. & 19 & 59,4 & 12 & 37,5 & 1 & 3,1 & 0 & 0 & 0 & 0 \\
\hline 7 & $\begin{array}{l}\text { Me informo de los beneficios de hacer } \\
\text { ejercicios. }\end{array}$ & 20 & 62,5 & 11 & 34,4 & 0 & 0 & 0 & 0 & 1 & 3,1 \\
\hline 8 & $\begin{array}{l}\text { Reconozco que hago más de lo que } \\
\text { debería de hacer en cuanto a mi rutina. }\end{array}$ & 9 & 28,1 & 10 & 31,3 & 3 & 9,4 & 8 & 25,0 & 2 & 6,3 \\
\hline 9 & He pensado en dejar de asistir al gimnasio. & 1 & 3,1 & 1 & 3,1 & 7 & 21,9 & 12 & 37,5 & 11 & 34,4 \\
\hline 10 & $\begin{array}{l}\text { Soy abusivo/a al momento de realizar mi } \\
\text { rutina del gimnasio. }\end{array}$ & 8 & 25,0 & 10 & 31,3 & 7 & 21,9 & 3 & 9,4 & 4 & 12,5 \\
\hline 11 & $\begin{array}{l}\text { Asistir al gimnasio ha cambiado mi forma } \\
\text { de ser. }\end{array}$ & 17 & 53,1 & 9 & 28,1 & 4 & 12,5 & 1 & 3,1 & 1 & 3,1 \\
\hline 12 & $\begin{array}{l}\text { Prefiero ir al gimnasio que compartir } \\
\text { tiempo con amigos familia o parejas. }\end{array}$ & 2 & 6,3 & 10 & 31,3 & 10 & 31,3 & 6 & 18,8 & 4 & 12,5 \\
\hline 13 & $\begin{array}{l}\text { Pienso que las personas de mi entorno son } \\
\text { mejores que yo. }\end{array}$ & 4 & 12,5 & 5 & 15,6 & 8 & 25,0 & 8 & 25,0 & 7 & 21,9 \\
\hline
\end{tabular}

La presente tabla muestra los resultados de los 13 ítems más importantes en este estudio y su gran porcentaje, donde se demuestran las actitudes que tienen los y las adolescentes, cabe destacar que los porcentajes mayores para estas son: El ítem Realizo más de 2 horas de rutina equivalente a un 62,5\%, igualándose esta cantidad con el ítem, me informo de los beneficios de hacer ejercicios físicos con un 62,5\%, a esta cantidad le sigue el ítem, Estoy dispuesto a asistir todo el día al gimnasio, con $59,4 \%$ y el ítem, Realizo rutina en el gimnasio con un $59,4 \%$, para el ítem, siento que necesito ir al gimnasio con un 56,3\%, siendo el ítem, Asistir al gimnasio ha cambiado mi forma de ser con un 53,1\%, siguiendo este con el ítem, Realizo mis rutinas moderadamente con un $37,5 \%$, igualándose en cantidades del ítem, He pensado en dejar de asistir al gimnasio con un $37,5 \%$, el ítem, Reconozco que hago más de lo que debería de hacer en cuanto a mi rutina con un 31,3\% igualándose en cantidades con el Ítem, Soy abusivo/a al momento de realizar mi rutina del gimnasio con un $31,3 \%$ y el ítem, Prefiero ir al gimnasio que compartir tiempo con amigos familia o parejas con un $31,3 \%$, siendo los últimos el ítem, Pienso que las personas de mi entorno son mejores que yo con un $25,0 \%$ al igual el ítem, comparo mi figura estando con otras personas con un $25,0 \%$.

Es notable que los pensamientos que tienen los adolescentes están entrelazados con las actitudes lo cual estas se ven afectadas, resaltando su presencia al practicar ejercicios físicos excesivos.

Incidencia del rol de género en la percepción de la imagen corporal de los adolescentes que practican ejercicios físicos excesivos

En los siguientes diagramas se muestran los resultados cuantitativos del listado libre, en cuanto a la incidencia del rol de género, tanto masculino, como femenino, los adolescentes asociaron palabras, en relación a la percepción de la imagen corporal. Cabe destacar que se tomaron las que más se frecuentaban para realizar este análisis, veremos a continuación quien incide más en cuanto al género. 


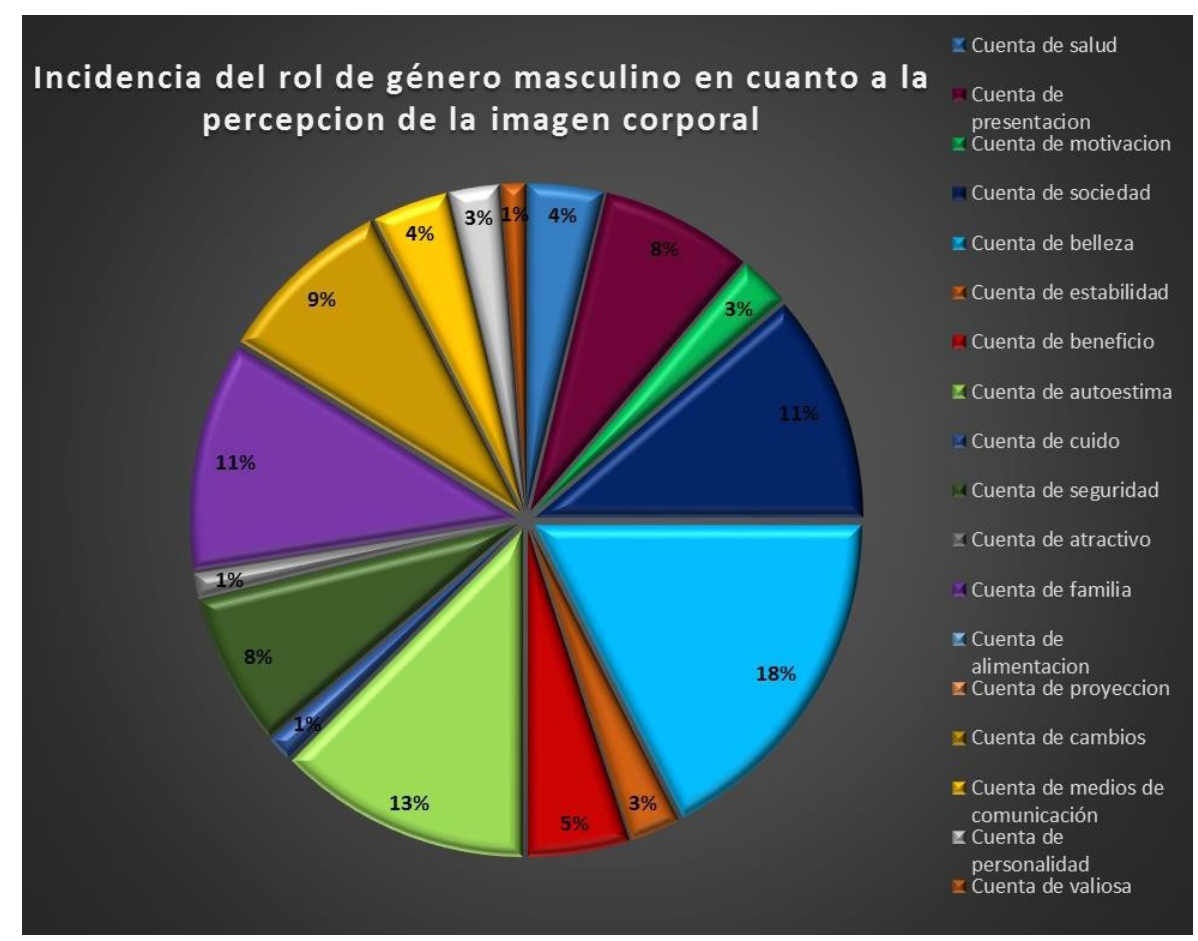

Análisis cuantitativo/incidencia del rol de género masculino

Las palabras que más predominan en los adolescentes acerca de la percepción que ellos tienen de su imagen corporal son con un $18 \%$ belleza, con un $13 \%$ autoestima, con un $11 \%$ familia e igual con un $11 \%$ sociedad, con un $9 \%$ cambios, con un $8 \%$ presentación e igual con un $8 \%$ seguridad, con un $5 \%$ beneficios, con un $4 \%$ medios de comunicación e igual con un $4 \%$ salud, con un $3 \%$ personalidad, con un $3 \%$ estabilidad, con un 3\% motivación, con un $1 \%$ valiosa, con un $1 \%$ cuido, con un $1 \%$ atractivo Vemos que las percepciones de los adolescentes acerca de lo que piensan de su imagen corporal sobresalen belleza y autoestima.

En el siguiente diagrama se muestran los resultados en relación a la incidencia del rol de género femenino

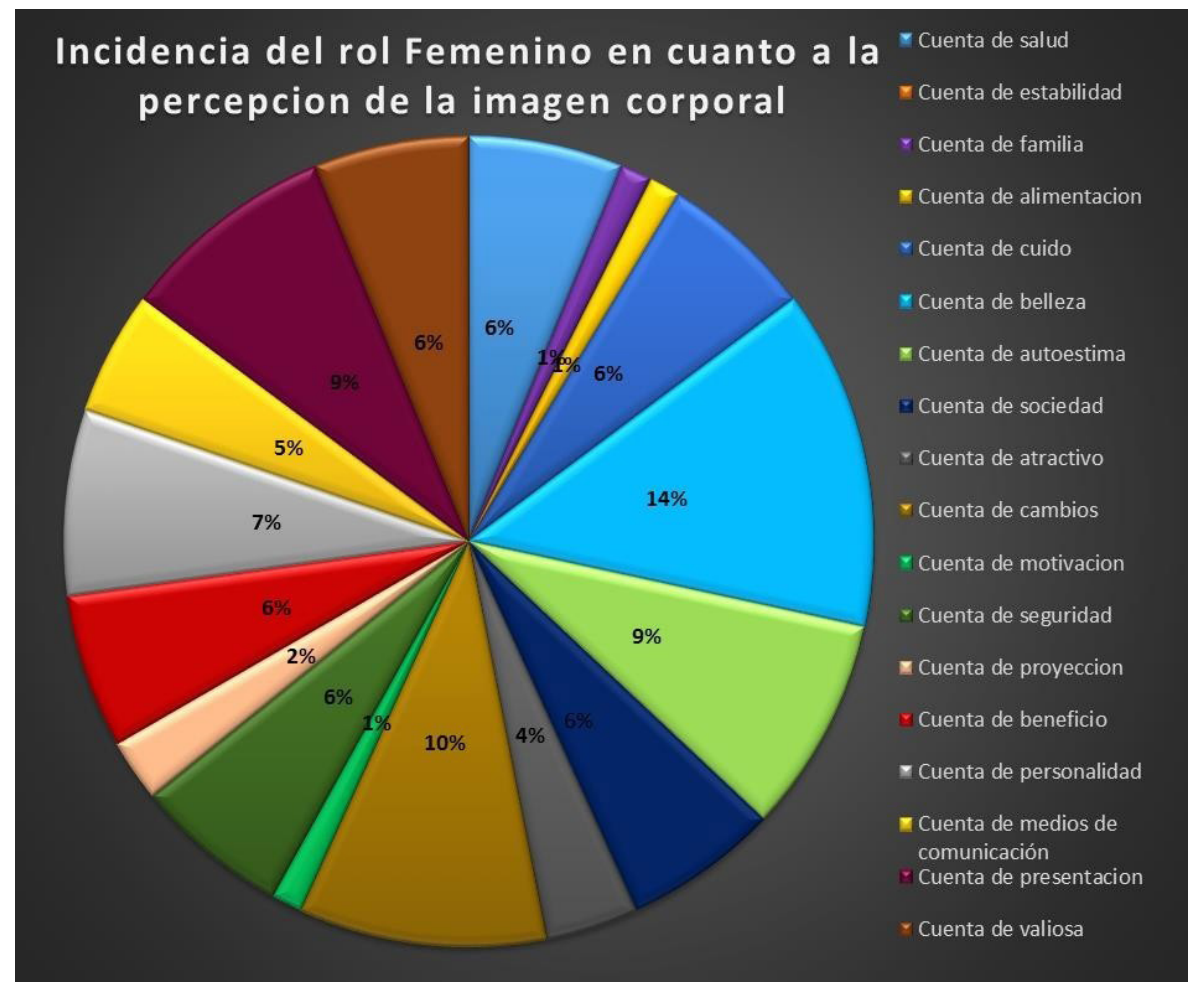


Análisis cuantitativo listado libre/Incidencia del rol de género femenino en la percepción de la imagen corporal

Las palabras más relevantes que muestran la percepción que tienen las adolescentes acerca de su imagen corporal son con un $14 \%$ belleza, con un $10 \%$ cambios, con un $9 \%$ presentación e igual con un $9 \%$ autoestima, seguidamente con un $7 \%$ personalidad, con un $6 \%$ valiosa e igual con un $6 \%$ beneficios, con un $6 \%$ seguridad, con un $6 \%$ sociedad, con un $6 \%$ cuido y con un $6 \%$ salud, con un $5 \%$ medios de comunicación, con $4 \%$ atractiva, con un $2 \%$ proyección, con un $1 \%$ familia e igual con un $1 \%$ alimentación y con un $1 \%$ motivación.

En los resultados anteriores se puede mostrar que existe mayor incidencia en el rol masculino que en el rol femenino, en cuanto a la percepción que estos tienen de su imagen corporal, cabe destacar que al ser encontrado este relevante resultado se rompen estereotipos en cuanto al género, porque socialmente estaba estipulado que existía más preocupación por el cuerpo en mujeres que en los hombres. Esto muestra que los adolescentes del género masculino podrían generar a un futuro la patología como lo es el Trastorno dismórfico corporal o también conocido como vigorexia; que esta es el deseo de obtener mayor masa muscular en el cuerpo, obteniendo mayor preocupación y asistiendo excesivamente a los gimnasios.

A través del análisis, se han constatado las teorías de los principales autores como: Erik Erikson, González, Niesser, Walter Lippmann, Santrock y Vernieri, que estos se aluden a la adolescencia, la percepción de la imagen corporal y a los ejercicios físicos excesivos.

\section{CONCLUSIONES}

El principal hallazgo fue, que las percepciones que poseen los adolescentes a cerca de la imagen corporal, se basan en aquella preocupación por verse bien, porque piensan que el físico es importante para la vida diaria, aludiendo que se sienten satisfechos logrando lo que ellos quieren en cuanto a su apariencia.

A los adolescentes les importa la opinión que tienen los demás acerca de su apariencia y que tanto los medios de comunicación como la sociedad, muestran una imagen corporal estereotipada de lo que deberían ser un hombre o una mujer en cuanto a la belleza. Es por ello que estos asisten a los gimnasios para modificar su cuerpo, además, siendo reforzados externamente por su familia y amigos, el cual es donde primeramente quieren ser aceptados.

Por otra parte, los adolescentes retoman actitudes que en su mayoría son negativas, recordando que estos descuidan ámbitos de su vida como lo es la familia, escuela, amigos y pareja, además estos expresan que están dispuestos a asistir todo el día al gimnasio porque este método ha cambiado su forma de ser, cabe destacar que los mismos proceden a realizar rutinas en el gimnasio que sobrepasan las dos horas.

Se realizó una comparación en la cual esta alude a que existe una mayor incidencia en el rol masculino que en el rol femenino, en cuanto a la percepción que estos tienen de su imagen corporal, cabe destacar que al ser encontrado este relevante resultado se rompen estereotipos en cuanto al género, porque socialmente estaba estipulado que existía más preocupación por el cuerpo en mujeres que en los hombres.

Esto muestra que los adolescentes del género masculino podrían generar a un futuro la patología como lo es el Trastorno dismórfico corporal o también conocido como vigorexia; esta se basa en el deseo de generar mayor masa muscular en el cuerpo, obteniendo mayor preocupación y asistiendo excesivamente a los gimnasios.

De igual manera los adolescentes entre 14 y 17 años se ven influenciados por la percepción de la imagen corporal y esto los lleva a mejorar y perfeccionar su cuerpo, asistiendo a los gimnasios y al no ver esos cambios que desean, asisten excesivamente a los mismos, tratando de arreglar algo que no está más que en su mente, debido a que existe una distorsión cognitiva de la imagen corporal.

A partir de lo encontrado se elaboró como sugerencia un plan de intervención psicológica para prevenir el trastorno dismórfico corporal o también llamado vigorexia, esto será para que los próximos investigadores sigan la línea de investigación. 


\section{AGRADECIMIENTOS}

A los adolescentes participantes de esta investigación, quienes brindaron su apoyo a la contribución de este estudio mediante una participación activa, también a los dueños de los gimnasios quienes permitieron formalmente aplicar instrumentos de recolección de datos. A MS.c. Franklin Solís Zúniga quien, como tutor del estudio investigativo, demostró Interés a sus estudiantes, brindándoles un acompañamiento, donde expresaba como se realizaría todo el proceso y sin duda alguna nuestra universidad UNANManagua, FAREM-Estelí, que nos permite crecer como estudiantes y seres humanos, conociendo sobre temáticas nuevas y llenándonos de aprendizaje.

\section{REFERENCIAS}

Arróliga Paz, G., López Moreno, R., \& Rodríguez Moreno, B. (2010). Factores Psicosociales asociados a la aparicion de conductas vigorexicas en los jovenes que asisten a los gimnacios de la ciudad de Estelí. Estelí.

Aviles, N. (2013 - 2015). Estudio de la satisfaccion de la imagen corporal en la educacion primaria, relaciones con la actividad fisica $y$ nivel de condicion de los escolares. Malaga España.

Balbi, M. B. (2013). Universidad del Salvador, Buenos Aires. Buenos Aires: USAL. Obtenido de http:// www.di.usaf- edu.ar $>$ balbia-ma.-belén.pdf

Kishory, C. (2010). Manifestaciones del trastorno vigorexia presentes en usuarios de gimnasios del municipio libertador del edo Mérida.

Vinueza, A. B. (Febrero de 2015). Prevalencia de conductas alimentarias de riesgo y trastornos alimentarios en adultos y jóvenes deportistas. 